\title{
A Potential Neuroanatomical Dissociation of DARPP-32 in Striatal ERK Signaling
}

\author{
Kristin B. Dupre \\ Behavioral Neuroscience Program, Department of Psychology, State University of New York at Binghamton, Binghamton, New York $13902-6000$ \\ Review of Gerfen et al. (http://www.jneurosci.org/cgi/content/full/28/28/7113)
}

Two main functions of the dopamine system involve reward and movement, and as such, dopamine modulation is central to the disorders of drug addiction and Parkinson's disease (PD). The striatum, a key target of dopamine pathways, plays an essential role in these functions. The dorsal striatum, comprised of the caudate nucleus and putamen, is involved in movement and receives its dopaminergic input mainly from the substantia nigra pars compacta. The ventral striatum, consisting of the nucleus accumbens shell and core, is involved in the reward pathway and receives its dopaminergic input mainly from the ventral tegmental area. Both dorsal and ventral striata also receive glutamatergic input from the cerebral cortex, which conveys context-dependent information about ongoing behavior (Seger and Cincotta, 2006). Dopamine modulates plasticity at glutamatergic corticostriatal synapses, and this regulation is an integral mechanism of dopaminecontrolled learning (Reynolds and Wickens, 2002).

Both psychostimulant addiction (Valjent et al., 2005) and L-DOPAinduced dyskinesia (LID), a common side

Received Sept. 1, 2008; revised Sept. 26, 2008; accepted Sept. 29, 2008.

I would like to thank my mentor, Dr. Christopher Bishop, my committee members, Dr. Lisa Savage and Dr. Terrence Deak, and my labmates, Christopher J. Barnum, Karen L. Eskow, and Corinne Y. Ostock.

Correspondence should be addressed to Kristin B. Dupre, Behavioral Neuroscience Program, Department of Psychology, State University of New York at Binghamton, 4400 Vestal Parkway East, Binghamton, NY 13902. E-mail: kdupre1@binghamton.edu.

DOI:10.1523/JNEUROSCI.4160-08.2008

Copyright $\odot 2008$ Society for Neuroscience $\quad 0270-6474 / 08 / 2810783-03 \$ 15.00 / 0$ effect of chronic dopamine replacement therapy in PD (Santini et al., 2007), involve striatal synaptic plasticity at glutamatergic synapses, which depends on the activation of extracellular signalregulated kinase 1/2 (ERK1/2). Figure 1 summarizes the signaling pathways proposed by Valjent et al. (2005) and Santini et al. (2007) in the activation of ERK1/2. Dopamine $D_{1}$ receptor activation of protein kinase A (PKA) phosphorylates mitogen-activated protein kinase kinase (MEK) to activate ERK1/2. PKA also regulates dopamine- and cAMP-dependent phosphoprotein of $32 \mathrm{kDa}$ (DARPP-32), which is selectively expressed in medium spiny neurons. DARPP-32 may regulate ERK1/2 by inhibiting PP-1, which normally acts to inhibit ERK1/2. Glutamate activation of NMDA receptors, through $\mathrm{Ca}^{2+} /$ calmodulin-regulated pathways, also phosphorylates MEK to activate ERK1/2. The phosphorylated ERK1/2 translocates to nuclei of medium spiny neurons and leads to changes in protein expression.

Psychostimulants, by indirectly acting on $\mathrm{D}_{1}$ receptors, have been shown to amplify NMDA receptor-mediated activation of ERK1/2 (Valjent et al., 2005). This activation and associated behaviors are blocked by $D_{1}$ receptor antagonists, NMDA receptor antagonists, and deletion of DARPP-32 genes (Valjent et al., 2005), implicating DARPP-32 as integral to $\mathrm{D}_{1}$ and NMDA-mediated signaling that evokes plasticity involved in psychostimulant addiction. Additionally, in dopamine-depleted PD models, there is robust activation of ERK $1 / 2$ by $D_{1}$ receptor agonists in neurons of the dopaminedepleted dorsal striatum (Gerfen et al., 2002). More recently, Santini et al. (2007) suggested that the interplay between DARPP-32 and ERK1/2, like in psychostimulant addiction, was critical to the development of LID. In a recent issue of The Journal of Neuroscience, Gerfen et al. (2008) extend these previous findings and indicate a unique site-specific neuroanatomical dissociation of DARPP-32 from ERK1/2 signaling by showing that DARPP-32-dependent psychostimulantevoked activation of ERK1/2 in the ventral striatum may not be relevant to the dorsal striatal plasticity involved in LID.

The first set of experiments done by Gerfen et al. (2008) investigated the involvement of DARPP-32 on psychostimulant-induced activation of ERK1/2 in the dorsal and ventral striatum using immunohistochemistry. It was found that in wild-type mice, D-amphetamine or cocaine potently activated ERK1/2 in the nucleus accumbens, whereas only modest effects were found in medial regions of dorsal striatum [Gerfen et al. (2008), their Fig. 1 (http://www. jneurosci.org/cgi/content/full/28/28/ $7113 / \mathrm{F} 1)]$. In both $\mathrm{D}_{1}$ and DARPP-32 knock-out mice, amphetamine- and cocaine-induced activation of nucleus accumbens ERK1/2 was reduced (66 and $61 \%$, respectively). In stark contrast, neither $\mathrm{D}_{1}$ nor DARPP-32 knock-out impacted ERK1/2 activation in the dorsal 
striatum. Therefore, it was suggested that activation of ERK1/2 through DARPP32-mediated mechanisms in the ventral, but not dorsal, striatum might be critical to the plasticity involved in psychostimulant drug addiction.

To advance their findings to PDrelated plasticity, Gerfen et al. (2008) next examined the role of DARPP-32 in $\mathrm{D}_{1}$ agonist- and L-DOPA-induced activation of ERK1/2. Wild-type and DARPP-32 knock-out mice were rendered hemiparkinsonian with unilateral lesions of the nigrostriatal dopamine system. Immunohistochemistry indicated that while there was little activation of ERK1/2 in the intact dorsal striatum, treatment with either the $D_{1}$ agonist SKF81297 (6-chloro-7,8dihyroxy-1-phenyl-2,3,4,5-tetrahydro$1 \mathrm{H}$-3-bezazepine-hydrobromide) or LDOPA significantly activated ERK1/2 in the dopamine-depleted striatum of both wild-type and DARPP-32 knock-out mice [Gerfen et al. (2008), their Fig. 4 (http:// www.jneurosci.org/cgi/content/full/28/ $28 / 7113 / F 4)]$. Finally, to confirm this, Gerfen et al. (2008) used Western blot techniques to show that L-DOPA increased ERK2 in the dopamine-depleted striatum of both wild-type and DARPP-32 knock-out mice [Gerfen et al. (2008), their Fig. 5 (http://www.jneurosci.org/ cgi/content/full/28/28/7113/F5)]. In all, it was concluded that $\mathrm{D}_{1}$ agonist and $\mathrm{L}$ DOPA treatment activate ERK1/2 in the dopamine-depleted (but not intact) dorsal striatum, and that though this activation is dependent on $D_{1}$ receptor stimulation [Gerfen et al. (2008), their supplemental Fig. 2 (http://www.jneurosci.org/ cgi/content/full/28/28/7113/DC1)], it is not DARPP-32 dependent.

Although Gerfen et al. (2008) found clear differences in the dependence of DARPP-32 between the dorsal and ventral striatum in psychostimulant-induced activation of ERK1/2, it is interesting to note that there was no impact of DARPP-32 between the dorsal and ventral striatum after $\mathrm{D}_{1}$ agonist and L-DOPA administration [Gerfen et al. (2008), their Table 3 (http://www.jneurosci.org/cgi/content/ full/28/28/7113/T3) and Table 4 (http:// www.jneurosci.org/cgi/content/full/28/ 28/7113/T4)]. This anatomical difference may be due to the divergent pharmacological actions of these various compounds. Cocaine and amphetamine act on the dopamine transporter and use endogenous dopamine, whereas $\mathrm{D}_{1}$ agonists directly stimulate the receptor and LDOPA provides exogenous dopamine through its conversion. Psychostimulant

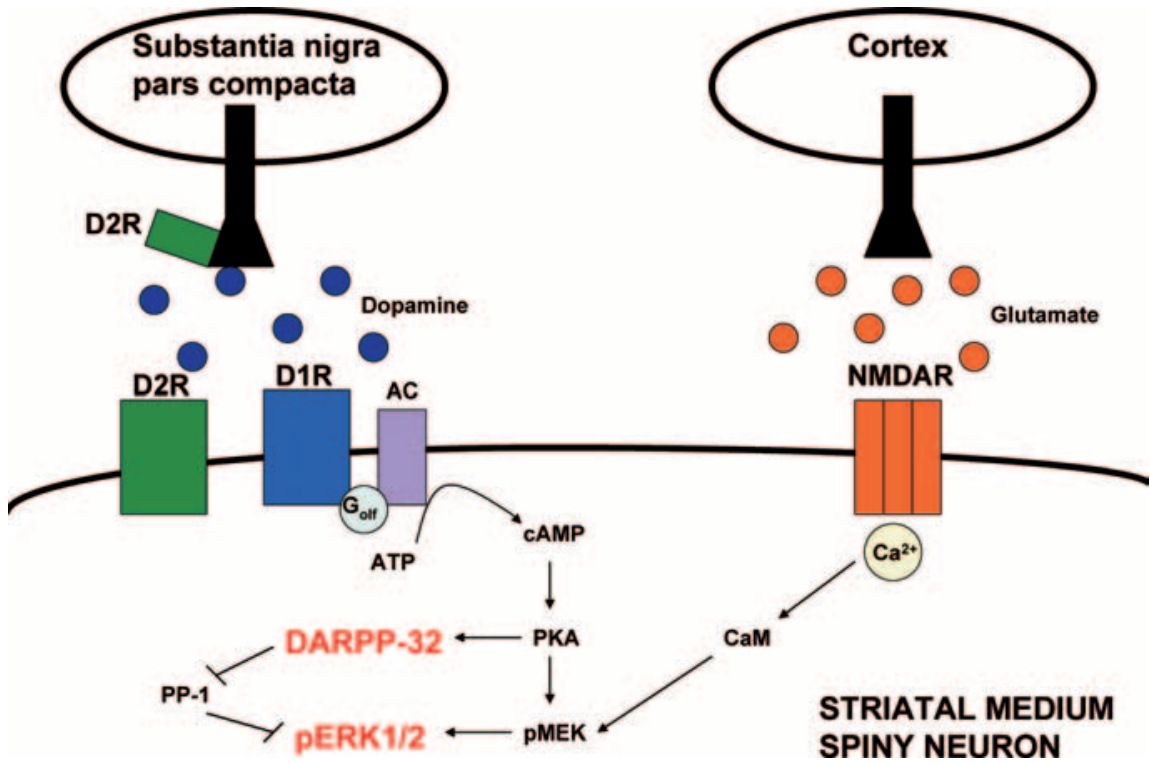

Figure 1. Schematic diagram illustrating the possible involvement of DARPP-32 and ERK1/2 in striatal synaptic plasticity. Glutamatergic inputs from the cortex stimulate NMDA receptors (NMDARs), while dopamine $D_{1}$ receptors (D1Rs) and $D_{2}$ receptors $(D 2 R)$ receive dopaminergic input from the substantia nigra pars compacta. Administration of psychostimulants, $D_{1}$ receptor agonists, or $L-D O P A$ leads to $D_{1}$ receptor, $G_{\text {olf }}$ protein coupled-activation of adenylyl cyclase. This, in turn, leads to the production of cAMP, which activates PKA. PKA-mediated regulation of DARPP-32 and phosphorylation of MEK (pMEK) influence the phosphorylation of ERK1/2 (pERK1/2). DARPP-32 is thought to regulate ERK1/2 by inhibiting protein phosphatase 1 (PP-1), which

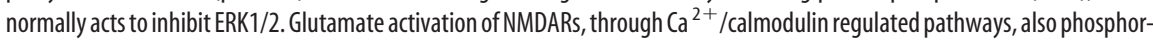
ylates MEK to activate ERK1/2. The phosphorylated ERK1/2 translocates to the nucleus of the medium spiny neuron and leads to changes in protein expression. Activation of these pathways is implicated in the long-term changes and striatal synaptic plasticity that is involved in psychostimulant addiction and L-DOPA-induced dyskinesia in Parkinson's disease.

activation of ERK1/2 in the ventral striatum depended on DARPP-32 [Gerfen et al. (2008), their Fig. 1 (http://www. jneurosci.org/cgi/content/full/28/28/ $7113 / \mathrm{F} 1)]$. If $\mathrm{D}_{1}$ agonist and L-DOPA acted in the same manner as amphetamine and cocaine, then a significant decrease $(\sim 61 \%)$ in ERK $1 / 2$ activation would have been predicted to occur in the dopamine-intact ventral striatum of DARPP-32 knock-out mice in Gerfen et al. (2008), Table 3 (http://www.jneurosci. org/cgi/content/full/28/28/7113/T3) and Table 4 (http://www.jneurosci.org/cgi/ content/full/28/28/7113/T4). This was not the case, and thus, the inherent differences in the mechanisms of action of the compounds tested may contribute in part to the different relationship of DARPP-32 and ERK1/2 in the dorsal and ventral striatum.

Insofar as the present investigation clearly extends knowledge regarding the role of DARPP-32 and ERK1/2 signaling in the striatum, the results are contrary to previous work. For example, Valjent et al. (2005) showed that DARPP-32 phosphorylation is required for activation of ERK by cocaine in both the dorsal and ventral striatum and that locomotor sensitization to cocaine was blocked in DARPP-32 knock-out mice. Santini et al.
(2007) emphasized the importance between high and low dyskinetic mice in the activation of ERK1/2. In their study, DARPP-32 knock-out mice had lower axial, forelimb, and orolingual abnormal involuntary movements after $10 \mathrm{~d}$ of L-DOPA treatment than those of wildtype mice. In addition, Santini et al. (2007) found less L-DOPA-induced ERK1/2 activation in the DARPP-32 knock-out mice. Although it is difficult to discern the reasons for these discrepancies, both Valjent et al. (2005) and Santini et al. (2007) used convergent behavioral measures. Application of behavioral variables in future investigations is clearly paramount.

The study by Gerfen et al. (2008) provides strong evidence for the role of $\mathrm{D}_{1}$ and DARPP-32 in the activation of ERK1/2 by psychostimulant administration, while implicating divergent roles between the dorsal and ventral striatum in these effects. As such, several questions arise. First, do different psychostimulants activate ERK differently in the dorsal and ventral striatum and what is the role of DARPP-32 in this neuroanatomical difference? Second, with regard to the $\mathrm{PD}$ model, what is the role of the $\mathrm{D}_{2}$ receptor? Although $\mathrm{D}_{1}$ receptors may be more important in the signaling mechanisms that 
occur after DA depletion (Gerfen et al., 2002; Aubert et al., 2005), $\mathrm{D}_{2}$ receptor agonists have been shown to activate ERK $1 / 2$ in the dorsal striatum (Cai et al., 2000; Gerfen et al., 2002). Third, does DARPP-32 have an influential role in ERK1/2 activation in only those animals that become dyskinetic? The discrepancy between Santini et al. (2007) and Gerfen et al. (2008) may be attributable to effects on behavior. Finally, given recent research investigating the connectivity between the dorsal and ventral striatum in cocaineseeking behavior (Belin and Everitt, 2008), what do the neuroanatomical differences between psychostimulant- and $\mathrm{D}_{1}$ agonist/L-DOPA-induced activation of ERK1/2 mean with regard to the interaction between the dorsal and ventral striatum? The recent study by Gerfen et al. (2008) has opened the door to explore and answer these challenging questions, which would provide beneficial insight to the understanding of the neural mechanisms involved in the plasticity of psychostimulant drug addiction and LID in PD.

\section{References}

Aubert I, Guigoni C, Håkansson K, Li Q, Dovero S, Barthe N, Bioulac BH, Gross CE, Fisone G, Bloch B, Bezard E (2005) Increased D1 dopamine receptor signaling in levodopainduced dyskinesia. Ann Neurol 57:17-26.

Belin D, Everitt BJ (2008) Cocaine seeking habits depend on dopamine-dependent serial connectivity linking the ventral with the dorsal striatum. Neuron 57:432-441.

Cai G, Zhen X, Uryu K, Friedman E (2000) Activation of extracellular signal-regulated protein kinase is associated with a sensitized locomotor response to $\mathrm{D}(2)$ dopamine receptor stimulation in unilateral 6-hydroxydopamine-lesioned rats. J Neurosci 20:1849-1857.

Gerfen CR, Miyachi S, Paletzki R, Brown P (2002) $D_{1}$ dopamine receptor supersensitivity in the dopamine-depleted striatum results from a switch in the regulation of ERK1/2/ MAP kinase. J Neurosci 22:5042-5054.

Gerfen CR, Paletzki R, Worley P (2008) Dif- ferences between dorsal and ventral striatum in drdla dopamine receptor coupling of dopamine- and cAMP-regulated phosphoprotein-32 to activation of extracellular signal-regulated kinase. J Neurosci 28:7113-7120.

Reynolds JN, Wickens JR (2002) Dopaminedependent plasticity of corticostriatal synapses. Neural Netw 15:507-521.

Santini E, Valjent E, Usiello A, Carta M, Borgkvist A, Girault JA, Hervé D, Greengard P, Fisone G (2007) Critical involvement of cAMP/ DARPP-32 and extracellular signal-regulated protein kinase signaling in L-DOPA-induced dyskinesia. J Neurosci 27:6995-7005.

Seger CA, Cincotta CM (2006) Dynamics of frontal, striatal, and hippocampal systems during rule learning. Cereb Cortex 16:1546-1555.

Valjent E, Pascoli V, Svenningsson P, Paul S, Enslen H, Corvol JC, Stipanovich A, Caboche J, Lombroso PJ, Nairn AC, Greengard P, Hervé D, Girault JA (2005) Regulation of a protein phosphatase cascade allows convergent dopamine and glutamate signals to activate ERK in the striatum. Proc Natl Acad Sci U S A 102: 491-496. 